\title{
Multivariate Spectral Gradient Algorithm for Nonsmooth Convex Optimization Problems
}

\author{
Yaping $\mathrm{Hu}$ \\ School of Science, East China University of Science and Technology, Shanghai 200237, China \\ Correspondence should be addressed to Yaping Hu; yapinghu@163.com
}

Received 20 April 2015; Revised 4 July 2015; Accepted 5 July 2015

Academic Editor: Dapeng P. Du

Copyright (C) 2015 Yaping Hu. This is an open access article distributed under the Creative Commons Attribution License, which permits unrestricted use, distribution, and reproduction in any medium, provided the original work is properly cited.

We propose an extended multivariate spectral gradient algorithm to solve the nonsmooth convex optimization problem. First, by using Moreau-Yosida regularization, we convert the original objective function to a continuously differentiable function; then we use approximate function and gradient values of the Moreau-Yosida regularization to substitute the corresponding exact values in the algorithm. The global convergence is proved under suitable assumptions. Numerical experiments are presented to show the effectiveness of this algorithm.

\section{Introduction}

Consider the unconstrained minimization problem

$$
\min _{x \in \Re^{n}} f(x),
$$

where $f: \mathfrak{R}^{n} \rightarrow \mathfrak{R}$ is a nonsmooth convex function. The Moreau-Yosida regularization [1] of $f$ at $x \in \mathfrak{R}^{n}$ associated with $z \in \mathfrak{R}^{n}$ is defined by

$$
F(x)=\min _{z \in \Re^{n}}\left\{f(z)+\frac{1}{2 \lambda}\|z-x\|^{2}\right\},
$$

where $\|\cdot\|$ is the Euclidean norm and $\lambda$ is a positive parameter. The function minimized on the right-hand side is strongly convex and differentiable, so it has a unique minimizer for every $z \in \mathfrak{R}^{n}$. Under some reasonable conditions, the gradient function of $F(x)$ can be proved to be semismooth $[2,3]$, though generally $F(x)$ is not twice differentiable. It is widely known that the problem

$$
\min _{x \in \Re^{n}} F(x)
$$

and the original problem (1) are equivalent in the sense that the two corresponding solution sets coincidentally are the same. The following proposition shows some properties of the Moreau-Yosida regularization function $F(x)$.
Proposition 1 (see Chapter XV, Theorem 4.1.4, [1]). The Moreau-Yosida regularization function $F$ is convex, finitevalued, and differentiable everywhere with gradient

$$
g(x) \equiv \nabla F(x)=\frac{1}{\lambda}(x-p(x)),
$$

where

$$
p(x)=\arg \min _{z \in \Re^{n}}\left\{f(z)+\frac{1}{2 \lambda}\|z-x\|^{2}\right\}
$$

is the unique minimizer in (2). Moreover, for all $x, y \in \mathfrak{R}^{n}$, one has

$$
\|g(x)-g(y)\| \leq \frac{1}{\lambda}\|x-y\|
$$

This proposition shows that the gradient function $g$ : $\mathfrak{R}^{n} \rightarrow \mathfrak{R}^{n}$ is Lipschitz continuous with modulus $1 / \lambda$. In this case, the gradient function $g$ is differentiable almost everywhere by the Rademacher theorem; then the Bsubdifferential [4] of $g$ at $x \in \mathfrak{R}^{n}$ is defined by

$$
\partial_{B} g(x)=\left\{V \in \mathfrak{R}^{n \times n}: V=\lim _{x_{k} \rightarrow x} \nabla g\left(x_{k}\right), x_{k} \in D_{g}\right\},
$$

where $D_{g}=\left\{x \in \mathfrak{R}^{n}: g\right.$ is differentiable at $\left.x\right\}$, and the next property of BD-regularity holds [4-6]. 
Proposition 2. If $g$ is BD-regular at $x$, then

(i) all matrices $V \in \partial_{B} g(x)$ are nonsingular;

(ii) there exists a neighborhood $\mathcal{N}$ of $x \in \mathfrak{R}^{n}, \kappa_{1}>0$, and $\kappa_{2}>0$; for all $y \in \mathcal{N}$, one has

$$
\begin{aligned}
d^{T} V d \geq \kappa_{1}\|d\|^{2}, \quad\left\|V^{-1}\right\| \leq & \kappa_{2}, \\
& \forall d \in \Re^{n}, V \in \partial_{B} g(y) .
\end{aligned}
$$

Instead of the corresponding exact values, we often use the approximate value of function $F(x)$ and gradient $g(x)$ in the practical computation, because $p(x)$ is difficult and sometimes impossible to be solved precisely. Suppose that, for any $\varepsilon>0$ and for each $x \in \mathfrak{R}^{n}$, there exists an approximate vector $p^{a}(x, \varepsilon) \in \mathfrak{R}^{n}$ of the unique minimizer $p(x)$ in (2) such that

$$
f\left(p^{a}(x, \varepsilon)\right)+\frac{1}{2 \lambda}\left\|p^{a}(x, \varepsilon)-x\right\|^{2} \leq F(x)+\varepsilon .
$$

The implementable algorithms to find such approximate vector $p^{a}(x, \varepsilon) \in \mathfrak{R}^{n}$ can be found, for example, in $[7,8]$. The existence theorem of the approximate vector $p^{a}(x, \varepsilon)$ is presented as follows.

Proposition 3 (see Lemma 2.1 in [7]). Let $\left\{x_{k}\right\}$ be generated according to the formula

$$
x_{k+1}=x_{k}-\alpha_{k} v_{k}, \quad \text { for } k=1,2, \ldots,
$$

where $\alpha_{k}>0$ is a stepsize and $v_{k}$ is an approximate subgradient at $x_{k}$; that is,

$$
\begin{array}{r}
v_{k} \in \partial_{\varepsilon_{k}} f\left(x_{k}\right)=\left\{v \mid f(v)-\left\langle v, x_{k}\right\rangle \leq f\left(x_{k}\right)+\varepsilon_{k}\right\}, \\
\text { for } k=1,2, \ldots .
\end{array}
$$

(i) If $v_{k}$ satisfies

$$
v_{k} \in \partial f\left(x_{k+1}\right), \quad \text { for } k=1,2, \ldots,
$$

then (11) holds with

$$
\varepsilon_{k}=f\left(x_{k}\right)-f\left(x_{k+1}\right)-\alpha_{k}\left\|v_{k}\right\|^{2} \geq 0 .
$$

(ii) Conversely, if (11) holds with $\varepsilon_{k}$ given by (13), then (12) holds: $x_{k+1}=p^{a}\left(x_{k}, \varepsilon_{k}\right)$.

We use the approximate vector $p^{a}(x, \varepsilon)$ to define approximation function and gradient values of the Moreau-Yosida regularization, respectively, by

$$
\begin{aligned}
& F^{a}(x, \varepsilon)=f\left(p^{a}(x, \varepsilon)\right)+\frac{1}{2 \lambda}\left\|p^{a}(x, \varepsilon)-x\right\|^{2}, \\
& g^{a}(x, \varepsilon)=\frac{\left(x-p^{a}(x, \varepsilon)\right)}{\lambda} .
\end{aligned}
$$

The following proposition is crucial in the convergence analysis. The proof of this proposition can be found in [2].
Proposition 4. Let $\varepsilon$ be arbitrary positive number and let $p^{a}(x, \varepsilon)$ be a vector satisfying (9). Then, one gets

$$
\begin{aligned}
F(x) & \leq F^{a}(x, \varepsilon) \leq F(x)+\varepsilon, \\
\left\|g^{a}(x, \varepsilon)-g(x)\right\| & \leq \sqrt{\frac{2 \varepsilon}{\lambda}}, \\
\left\|p^{a}(x, \varepsilon)-p(x)\right\| & \leq \sqrt{2 \lambda \varepsilon} .
\end{aligned}
$$

Algorithms which combine the proximal techniques with Moreau-Yosida regularization for solving the nonsmooth problem (1) have been proved to be effective $[7,9,10]$, and also some trust region algorithms for solving (1) have been proposed in $[5,11,12]$, and so forth. Recently, Yuan et al. [13, 14] and $\mathrm{Li}$ [15] have extended the spectral gradient method and conjugate gradient-type method to solve (1), respectively.

Multivariate spectral gradient (MSG) method was first proposed by Han et al. [16] for optimization problems. This method has a nice property that it converges quadratically for objective function with positive definite diagonal Hessian matrix [16]. Further studies on such method for nonlinear equations and bound constrained optimization can be found, for instance, in $[17,18]$. By using nonmonotone technique, some effective spectral gradient methods are presented in $[13,16,17,19]$. In this paper, we extend the multivariate spectral gradient method by combining with a nonmonotone line search technique as well as the Moreau-Yosida regulation function to solve the nonsmooth problem (1) and do some numerical experiments to test its efficiency.

The rest of this paper is organized as follows. In Section 2, we propose multivariate spectral gradient algorithm to solve (1). In Section 3, we prove the global convergence of the proposed algorithm; then some numerical results are presented in Section 4. Finally, we have a conclusion section.

\section{Algorithm}

In this section, we present the multivariate spectral gradient algorithm to solve the nonsmooth convex unconstrained optimization problem (1). Our approach is using the tool of the Moreau-Yosida regularization to smoothen the nonsmooth function and then make use of the approximate values of function $F$ and gradient $g$ in multivariate spectral gradient algorithm.

We first recall the multivariate spectral gradient algorithm [16] for smooth optimization problem:

$$
\min \left\{f(x) \mid x \in \mathfrak{R}^{n}\right\},
$$

where $f: \mathfrak{R}^{n} \rightarrow \mathfrak{R}$ is continuously differentiable and its gradient is denoted by $g$. Let $x_{k}$ be the current iteration; multivariate spectral gradient algorithm is defined by

$$
x_{k+1}=x_{k}-\operatorname{diag}\left\{\frac{1}{\lambda_{k}^{1}}, \frac{1}{\lambda_{k}^{2}}, \ldots, \frac{1}{\lambda_{k}^{n}}\right\} g_{k},
$$

where $g_{k}$ is the gradient vector of $f$ at $x_{k}$ and $\operatorname{diag}\left\{\lambda_{k}^{1}, \lambda_{k}^{2}\right.$, $\left.\ldots, \lambda_{k}^{n}\right\}$ is solved by minimizing

$$
\left\|\operatorname{diag}\left\{\lambda^{1}, \lambda^{2}, \ldots, \lambda^{n}\right\} s_{k-1}-u_{k-1}\right\|_{2}
$$


with respect to $\left\{\lambda^{i}\right\}_{i=1}^{n}$, where $s_{k-1}=x_{k}-x_{k-1}, u_{k-1}=g_{k}-$ $g_{k-1}$.

Denote the $i$ th element of $s_{k}$ and $y_{k}$ by $s_{k}^{i}$ and $y_{k}^{i}$, respectively. We present the following multivariate spectral gradient (MSG) algorithm.

Algorithm 5. Set $x_{0} \in \Re^{n}, \sigma \in(0,1), \beta>0, \lambda>0, \gamma \geq 0$, $\delta>0, \rho \in[0,1], \epsilon \in(0,1), E_{0}=1$, and $\tau_{0} \in(0,1] ;\left\{\tau_{k}\right\}$ is a strictly decreasing sequence with $\lim _{k \rightarrow 0} \tau_{k}=0, k:=0$.

Step 1. Set $\varepsilon_{0}=\tau_{0}$. Calculate $F^{a}\left(x_{0}, \varepsilon_{0}\right)$ by (14) as well as $g^{a}\left(x_{0}, \varepsilon_{0}\right)$ by (15). Let $J_{0}=F^{a}\left(x_{0}, \varepsilon_{0}\right), d_{0}=-g^{a}\left(x_{0}, \varepsilon_{0}\right)$.

Step 2. Stop if $\left\|g^{a}\left(x_{k}, \varepsilon_{k}\right)\right\|=0$. Otherwise, go to Step 3 .

Step 3. Choose $\varepsilon_{k+1}$ satisfying $0<\varepsilon_{k+1} \leq \min \left\{\tau_{k}\right.$, $\left.\tau_{k}\left\|g^{a}\left(x_{k}, \varepsilon_{k}\right)\right\|^{2}\right\}$; find $\alpha_{k}$ which satisfies

$$
F^{a}\left(x_{k}+\alpha_{k} d_{k}, \varepsilon_{k+1}\right)-J_{k} \leq \sigma \alpha_{k} g^{a}\left(x_{k}, \varepsilon_{k}\right)^{T} d_{k},
$$

where $\alpha_{k}=\beta 2^{-i_{k}}$ and $i_{k}$ is the smallest nonnegative integer such that (22) holds.

Step 4. Let $x_{k+1}=x_{k}+\alpha_{k} d_{k}$. Stop if $\left\|g^{a}\left(x_{k+1}, \varepsilon_{k+1}\right)\right\|=0$.

Step 5. Update $J_{k+1}$ by the following formula:

$$
\begin{aligned}
E_{k+1} & =\rho E_{k}+1, \\
J_{k+1} & =\frac{\rho E_{k} J_{k}+F^{a}\left(x_{k}+\alpha_{k} d_{k}, \varepsilon_{k+1}\right)}{E_{k+1}} .
\end{aligned}
$$

Step 6. Compute the search direction $d_{k+1}$ by the following:

(a) If $y_{k}^{i} / s_{k}^{i}>0$, then set $\lambda_{k+1}^{i}=y_{k}^{i} / s_{k}^{i}$; otherwise set $\lambda_{k+1}^{i}=s_{k}^{T} y_{k} / s_{k}^{T} s_{k}$ for $i=1,2, \ldots, n$, where $y_{k}=$ $g^{a}\left(x_{k+1}, \varepsilon_{k+1}\right)-g^{a}\left(x_{k}, \varepsilon_{k}\right)+\gamma s_{k}, s_{k}=x_{k+1}-x_{k}$.

(b) If $\lambda_{k+1}^{i} \leq \epsilon$ or $\lambda_{k+1}^{i} \geq 1 / \epsilon$, then set $\lambda_{k+1}^{i}=\delta$ for $i=$ $1,2, \ldots, n$.

Let $d_{k+1}=-\operatorname{diag}\left\{1 / \lambda_{k+1}^{1}, 1 / \lambda_{k+1}^{2}, \ldots, 1 / \lambda_{k+1}^{n}\right\} g^{a}\left(x_{k+1}, \varepsilon_{k+1}\right)$.

Step 7. Set $k:=k+1$; go back to Step 2 .

Remarks. (i) The definition of $\varepsilon_{k+1}=o\left(\left\|g^{a}\left(x_{k}, \varepsilon_{k}\right)\right\|^{2}\right)$ in Algorithm 5, together with (15) and Proposition 3, deduces that

$$
\begin{aligned}
\varepsilon_{k+1} & =o\left(\left\|x_{k}-p^{a}\left(x_{k}, \varepsilon_{k}\right)\right\|^{2}\right)=o\left(\left\|x_{k}-x_{k+1}\right\|^{2}\right) \\
& =o\left(\alpha_{k}^{2}\left\|d_{k}\right\|^{2}\right) ;
\end{aligned}
$$

then, with the decreasing property of $\varepsilon_{k+1}$, the assumed condition $\varepsilon_{k}=o\left(\alpha_{k}^{2}\left\|d_{k}\right\|^{2}\right)$ in Lemma 7 holds.

(ii) From the nonmonotone line search technique (22), we can see that $J_{k+1}$ is a convex combination of the function value $F^{a}\left(x_{k+1}, \varepsilon_{k+1}\right)$ and $J_{k}$. Also $J_{k}$ is a convex combination of the function values $F^{a}\left(x_{k}, \varepsilon_{k}\right), \ldots, F^{a}\left(x_{1}, \varepsilon_{1}\right), F^{a}\left(x_{0}, \varepsilon_{0}\right)$ as $J_{0}=F^{a}\left(x_{0}, \varepsilon_{0}\right) \cdot \rho$ is a positive value that plays an important role in manipulating the degree of nonmonotonicity in the nonmonotone line search technique, with $\rho=0$ yielding a strictly monotone scheme and with $\rho=1$ yielding $J_{k}=C_{k}$, where

$$
C_{k}=\frac{1}{k+1} \sum_{i=0}^{k} F^{a}\left(x_{i}, \varepsilon_{i}\right)
$$

is the average function value.

(iii) From Step 6, we can obtain that

$$
\begin{gathered}
\min \left\{\epsilon, \frac{1}{\delta}\right\}\left\|g^{a}\left(x_{k}, \varepsilon_{k}\right)\right\| \leq\left\|d_{k}\right\| \\
\leq \max \left\{\frac{1}{\epsilon}, \frac{1}{\delta}\right\}\left\|g^{a}\left(x_{k}, \varepsilon_{k}\right)\right\| ;
\end{gathered}
$$

then there is a positive constant $\mu$ such that, for all $k$,

$$
g^{a}\left(x_{k}, \varepsilon_{k}\right)^{T} d_{k} \leq-\mu\left\|g^{a}\left(x_{k}, \varepsilon_{k}\right)\right\|^{2},
$$

which shows that the proposed multivariate spectral gradient algorithm possesses the sufficient descent property.

\section{Global Convergence}

In this section, we provide a global convergence analysis for the multivariate spectral gradient algorithm. To begin with, we make the following assumptions which have been given in $[5,12-14]$.

Assumption A. (i) $F$ is bounded from below.

(ii) The sequence $\left\{V_{k}\right\}, V_{k} \in \partial_{B} g\left(x_{k}\right)$, is bounded; that is, there exists a constant $M>0$ such that, for all $k$,

$$
\left\|V_{k}\right\| \leq M
$$

The following two lemmas play crucial roles in establishing the convergence theorem for the proposed algorithm. By using (26) and (27) and Assumption A, similar to Lemma 1.1 in [20], we can get the next lemma which shows that Algorithm 5 is well defined. The proof ideas of this lemma and Lemma 1.1 in [20] are similar, hence omitted.

Lemma 6. Let $\left\{F^{a}\left(x_{k}, \varepsilon_{k}\right)\right\}$ be the sequence generated by Algorithm 5. Suppose that Assumption A holds and $C_{k}$ is defined by (25). Then one has $F^{a}\left(x_{k}, \varepsilon_{k}\right) \leq J_{k} \leq C_{k}$ for all $k$. Also, there exists a stepsize $\alpha_{k}$ satisfying the nonmonotone line search condition.

Lemma 7. Let $\left\{\left(x_{k}, \varepsilon_{k}\right)\right\}$ be the sequence generated by Algorithm 5. Suppose that Assumption A and $\varepsilon_{k}=o\left(\alpha_{k}^{2}\left\|d_{k}\right\|^{2}\right)$ hold. Then, for all $k$, one has

$$
\alpha_{k} \geq m_{0}
$$

where $m_{0}>0$ is a constant.

Proof (Proof by Contradiction). Let $\alpha_{k}$ satisfy the nonmonotone Armijo-type line search (22). Assume on the contrary 
that $\liminf _{k \rightarrow \infty} \alpha_{k}=0$ does hold; then there exists a subsequence $\left\{\alpha_{k}\right\}_{K^{\prime}}$ such that $\alpha_{k} \rightarrow 0$ as $k \rightarrow \infty$. From the nonmonotone line search rule (22), $\alpha_{k}^{\prime}=\alpha_{k} / 2$ satisfies

$$
F^{a}\left(x_{k}+\alpha_{k}^{\prime} d_{k}, \varepsilon_{k+1}\right)-J_{k}>\sigma \alpha_{k}^{\prime} g^{a}\left(x_{k}, \varepsilon_{k}\right)^{T} d_{k}
$$

together with $F^{a}\left(x_{k}, \varepsilon_{k}\right) \leq J_{k} \leq C_{k}$ in Lemma 6, we have

$$
\begin{aligned}
& F^{a}\left(x_{k}+\alpha_{k}^{\prime} d_{k}, \varepsilon_{k+1}\right)-F^{a}\left(x_{k}, \varepsilon_{k}\right) \\
& \quad \geq F^{a}\left(x_{k}+\alpha_{k}^{\prime} d_{k}, \varepsilon_{k+1}\right)-J_{k}>\sigma \alpha_{k}^{\prime} g^{a}\left(x_{k}, \varepsilon_{k}\right)^{T} d_{k} .
\end{aligned}
$$

By (28) and (31) and Proposition 4 and using Taylor's formula, there is

$$
\begin{aligned}
\sigma \alpha_{k}^{\prime} g^{a}\left(x_{k}, \varepsilon_{k}\right)^{T} d_{k}< & F^{a}\left(x_{k}+\alpha_{k}^{\prime} d_{k}, \varepsilon_{k+1}\right) \\
& -F^{a}\left(x_{k}, \varepsilon_{k}\right) \\
\leq & F\left(x_{k}+\alpha_{k}^{\prime} d_{k}\right)-F\left(x_{k}\right)+\varepsilon_{k+1} \\
= & \alpha_{k}^{\prime} d_{k}^{T} g\left(x_{k}\right) \\
& +\frac{1}{2}\left(\alpha_{k}^{\prime}\right)^{2} d_{k}^{T} V\left(u_{k}\right) d_{k}+\varepsilon_{k+1} \\
\leq & \alpha_{k}^{\prime} d_{k}^{T} g\left(x_{k}\right)+\frac{M}{2}\left(\alpha_{k}^{\prime}\right)^{2}\left\|d_{k}\right\|^{2} \\
& +\varepsilon_{k+1},
\end{aligned}
$$

where $u_{k} \in\left(x_{k}, x_{k+1}\right)$. From (32) and Proposition 4, we have

$$
\begin{aligned}
\frac{\alpha_{k}}{2} & =\alpha_{k}^{\prime}>\left[\frac{\left(g^{a}\left(x_{k}, \varepsilon_{k}\right)-g\left(x_{k}\right)\right)^{T} d_{k}-(1-\sigma) g^{a}\left(x_{k}, \varepsilon_{k}\right)^{T} d_{k}-\varepsilon_{k+1} / \alpha_{k}^{\prime}}{\left\|d_{k}\right\|^{2}}\right] \frac{2}{M} \\
& \geq\left[\frac{\mu(1-\sigma)\left\|g^{a}\left(x_{k}, \varepsilon_{k}\right)\right\|^{2}-\sqrt{2 \varepsilon_{k} / \lambda}\left\|d_{k}\right\|-\varepsilon_{k} / \alpha_{k}^{\prime}}{\left\|d_{k}\right\|^{2}}\right] \frac{2}{M}=\left[\frac{\mu(1-\sigma)\left\|g^{a}\left(x_{k}, \varepsilon_{k}\right)\right\|^{2}}{\left\|d_{k}\right\|^{2}}-\frac{o\left(\alpha_{k}\right)}{\sqrt{\lambda}}-o\left(\alpha_{k}\right)\right] \frac{2}{M} \\
& \geq\left[\frac{\mu(1-\sigma)}{(\max \{1 / \epsilon, 1 / \delta\})^{2}}-\frac{o\left(\alpha_{k}\right)}{\sqrt{\lambda}}-o\left(\alpha_{k}\right)\right] \frac{2}{M},
\end{aligned}
$$

where the second inequality follows from (26), Part 3 in Proposition 4 , and $\varepsilon_{k+1} \leq \varepsilon_{k}$, the equality follows from $\varepsilon_{k}=$ $o\left(\alpha_{k}^{2}\left\|d_{k}\right\|^{2}\right)$, and the last inequality follows from (27). Dividing each side by $\alpha_{k}$ and letting $k \rightarrow \infty$ in the above inequality, we can deduce that

$$
\frac{1}{2} \geq \lim _{k \rightarrow \infty}\left(\frac{2 \mu(1-\sigma)}{(\max \{1 / \epsilon, 1 / \delta\})^{2} M}\right) \frac{1}{\alpha_{k}}=+\infty,
$$

which is impossible, so the conclusion is obtained.

By using the above lemmas, we are now ready to prove the global convergence of Algorithm 5.

Theorem 8. Let $\left\{x_{k}\right\}$ be generated by Algorithm 5 and suppose that the conditions of Lemma 7 hold. Then one has

$$
\lim _{k \rightarrow \infty}\left\|g\left(x_{k}\right)\right\|=0 ;
$$

sequence $\left\{x_{k}\right\}_{k=0}^{\infty}$ has accumulation point, and every accumulation point of $\left\{x_{k}\right\}_{k=0}^{\infty}$ is optimal solution of problem (1).

Proof. Suppose that there exist $\epsilon_{0}>0$ and $k_{0}>0$ such that

$$
\left\|g^{a}\left(x_{k}, \varepsilon_{k}\right)\right\| \geq \epsilon_{0}, \quad \forall k>k_{0} .
$$

From (22), (26), and (29), we get

$$
\begin{gathered}
F^{a}\left(x_{k}+\alpha_{k} d_{k}, \varepsilon_{k+1}\right)-J_{k} \leq \sigma \alpha_{k} g^{a}\left(x_{k}, \varepsilon_{k}\right)^{T} d_{k} \\
\quad \leq-\sigma \alpha_{k} \min \left\{\epsilon, \frac{1}{\delta}\right\}\left\|g^{a}\left(x_{k}, \varepsilon_{k}\right)\right\|^{2} \\
\quad \leq-\sigma m_{0} \epsilon_{0} \min \left\{\epsilon, \frac{1}{\delta}\right\}, \quad \forall k>k_{0} .
\end{gathered}
$$

Therefore, it follows from the definition of $J_{k+1}$ and (23) that

$$
\begin{aligned}
J_{k+1} & =\frac{\rho E_{k} J_{k}+F^{a}\left(x_{k}+\alpha_{k} d_{k}, \varepsilon_{k+1}\right)}{E_{k+1}} \\
& \leq \frac{\rho E_{k} J_{k}+J_{k}-\sigma m_{0} \epsilon_{0} \min \{\epsilon, 1 / \delta\}}{E_{k+1}} \\
& \leq J_{k}-\frac{\sigma m_{0} \epsilon_{0} \min \{\epsilon, 1 / \delta\}}{E_{k+1}} .
\end{aligned}
$$

By Assumption A, $F$ is bounded from below. Further by Proposition 4, $F\left(x_{k}\right) \leq F^{a}\left(x_{k}, \varepsilon_{k}\right)$ for all $k$, we see that $F^{a}\left(x_{k}, \varepsilon_{k}\right)$ is bounded from below. Together with $F^{a}\left(x_{k}, \varepsilon_{k}\right) \leq$ $J_{k}$ for all $k$ from Lemma 6 , it shows that $J_{k}$ is also bounded from below. By (38), we obtain

$$
\sum_{k=k_{0}}^{\infty} \frac{\sigma m_{0} \epsilon_{0} \min \{\epsilon, 1 / \delta\}}{E_{k+1}}<\infty
$$

On the other hand, the definition of $E_{k+1}$ implies that $E_{k+1} \leq$ $k+2$, and it follows that

$$
\begin{aligned}
& \sum_{k=k_{0}}^{\infty} \frac{\sigma m_{0} \epsilon_{0} \min \{\epsilon, 1 / \delta\}}{E_{k+1}} \geq \sum_{k=k_{0}}^{\infty} \frac{\sigma m_{0} \epsilon_{0} \min \{\epsilon, 1 / \delta\}}{k+2} \\
& \quad=+\infty .
\end{aligned}
$$


This is a contradiction. Therefore, we should have

$$
\lim _{k \rightarrow \infty}\left\|g^{a}\left(x_{k}, \varepsilon_{k}\right)\right\|=0
$$

From (17) in Proposition 4 together with $\varepsilon_{k}$ as $k \rightarrow \infty$, which comes from the definition of $\varepsilon_{k}$ and $\lim _{k \rightarrow 0} \tau_{k}=0$ in Algorithm 5, we obtain

$$
\lim _{k \rightarrow \infty}\left\|g\left(x_{k}\right)\right\|=0
$$

Set $x^{*}$ as an accumulation point of sequence $\left\{x_{k}\right\}_{k=0}^{\infty}$; there is a convergent subsequence $\left\{x_{k_{l}}\right\}_{l=0}^{\infty}$ such that

$$
\lim _{l \rightarrow \infty} x_{k_{l}}=x^{*}
$$

From (4) we know that $g\left(x_{k}\right)=\left(x_{k}-p\left(x_{k}\right)\right) / \lambda$. Consequently, (42) and (43) show that $x^{*}=p\left(x^{*}\right)$. Hence, $x^{*}$ is an optimal solution of problem (1).

\section{Numerical Results}

This section presents some numerical results from experiments using our multivariate spectral gradient algorithm for the given test nonsmooth problems which come from [21]. We also list the results of [14] (modified Polak-RibièrePolyak gradient method, MPRP) and [22] (proximal bundle method, PBL) to make a comparison with the result of Algorithm 5. All codes were written in MATLAB R2010a and were implemented on a PC with $2.8 \mathrm{GHz} \mathrm{CPU}, 2 \mathrm{~GB}$ of memory, and Windows 8. We set $\beta=\lambda=1, \sigma=0.9$, $\epsilon=10^{-10}$, and $\gamma=0.01$, and the parameter $\delta$ is chosen as

$$
\delta= \begin{cases}1 & \text { if }\left\|g^{a}\left(x_{k}, \varepsilon_{k}\right)\right\|>1, \\ \left\|g^{a}\left(x_{k}, \varepsilon_{k}\right)\right\|^{-1} & \text { if } 10^{-5} \leq\left\|g^{a}\left(x_{k}, \varepsilon_{k}\right)\right\| \leq 1, \\ 10^{-5} & \text { if }\left\|g^{a}\left(x_{k}, \varepsilon_{k}\right)\right\|<10^{-5} ;\end{cases}
$$

then we adopt the termination condition $\left\|g^{a}\left(x_{k}, \varepsilon_{k}\right)\right\| \leq$ $10^{-10}$. For subproblem (5), the classical PRP CG method (called subalgorithm) is used to solve it; the algorithm stops if $\left\|\partial f\left(x_{k}\right)\right\| \leq 10^{-4}$ or $f\left(x_{k+1}\right)-f\left(x_{k}\right)+\left\|\partial f\left(x_{k+1}\right)\right\|^{2}-\left\|\partial f\left(x_{k}\right)\right\|^{2} \leq$ $10^{-3}$ holds, where $\partial f\left(x_{k}\right)$ is the subgradient of $f(x)$ at the point $x_{k}$. The subalgorithm will also stop if the iteration number is larger than fifteen. In its line search, the Armijo line search technique is used and the step length is accepted if the search number is larger than five. Table 1 contains problem names, problem dimensions, and the optimal values.

The summary of the test results is presented in Tables 23, where "Nr." denotes the name of the tested problem, "NF" denotes the number of function evaluations, "NI" denotes the number of iterations, and " $f(x)$ " denotes the function value at the final iteration.
TABLE 1: Test problems.

\begin{tabular}{lccc}
\hline Nr. & Problems & Dim. & $f_{\text {ops }}(x)$ \\
\hline 1 & Rosenbrock & 2 & 0 \\
2 & Crescent & 2 & 0 \\
3 & CB2 & 2 & 1.9522245 \\
4 & CB3 & 2 & 2.0 \\
5 & DEM & 2 & -3 \\
6 & QL & 2 & 7.20 \\
7 & LQ & 2 & -1.4142136 \\
8 & Mifflin 1 & 2 & -1.0 \\
9 & Mifflin 2 & 2 & -1.0 \\
10 & Wolfe & 2 & -8.0 \\
11 & Rosen-Suzuki & 4 & -44 \\
12 & Shor & 5 & 22.600162 \\
\hline
\end{tabular}

The value of $\rho$ controls the nonmonotonicity of line search which may affect the performance of the MSG algorithm. Table 2 shows the results for different parameter $\rho$, as well as different values of the parameter $\tau_{k}$ ranging from $1 / 6(k+2)^{6}$ to $1 / 2 k^{2}$ on problem Rosenbrock, respectively. We can conclude from the table that the proposed algorithm works reasonably well for all the test cases. This table also illustrates that the value of $\rho$ can influence the performance of the algorithm significantly if the value of $\varepsilon$ is within a certain range, and the choice $\rho=0.75$ is better than $\rho=0$.

Then, we compare the performance of MSG to that of the algorithms MPRP and PBL. In this test, we fix $\tau_{k}=$ $1 / 2 k^{2}$ and $\rho=0.75$. To illustrate the performance of each algorithm more specifically, we present three comparison results in terms of number of iterations, number of function evaluations, and the final objective function value in Table 3.

The numerical results indicate that Algorithm 5 can successfully solve the test problems. From the number of iterations in Table 3, we see that Algorithm 5 performs best among these three methods, and the final function value obtained by Algorithm 5 is closer to the optimal function value than those obtained by MPRP and PBL. In a word, the numerical experiments show that the proposed algorithm provides an efficient approach to solve nonsmooth problems.

\section{Conclusions}

We extend the multivariate spectral gradient algorithm to solve nonsmooth convex optimization problems. The proposed algorithm combines a nonmonotone line search technique and the idea of Moreau-Yosida regularization. The algorithm satisfies the sufficient descent property and its global convergence can be established. Numerical results show the efficiency of the proposed algorithm. 
TABLE 2: Results on Rosenbrock with different $\rho$ and $\varepsilon$.

\begin{tabular}{|c|c|c|c|c|}
\hline$\tau_{k}$ & $\begin{array}{c}\rho=0 \\
\mathrm{NI} / \mathrm{NF} / f(x)\end{array}$ & Time & $\begin{array}{c}\rho=0.75 \\
\mathrm{NI} / \mathrm{NF} / f(x)\end{array}$ & Time \\
\hline $1 / 2 k^{2}$ & $30 / 46 / 1.581752 e-9$ & 1.794 & $29 / 30 / 7.778992 e-9$ & 1.076 \\
\hline $1 / 3(k+2)^{3}$ & $28 / 38 / 5.207744 e-9$ & 1.420 & $26 / 27 / 6.541087 e-9$ & 1.023 \\
\hline $1 / 4(k+2)^{4}$ & $29 / 37 / 1.502034 e-9$ & 1.388 & $27 / 28 / 5.112699 e-9$ & 1.030 \\
\hline $1 / 5(k+2)^{5}$ & $27 / 37 / 1.903969 e-9$ & 1.451 & $27 / 28 / 6.329141 e-9$ & 1.092 \\
\hline $1 / 6(k+2)^{6}$ & $27 / 36 / 4.859901 e-9$ & 1.376 & $27 / 28 / 6.073222 e-9$ & 1.025 \\
\hline
\end{tabular}

TABLE 3: Numerical results for MSG/MPRP/PBL on problems 1-12.

\begin{tabular}{lcccc}
\hline Nr. & MSG & MPRP & PBL & $f_{\text {ops }}(x)$ \\
\hline 1 & NI/NF/ $f(x)$ & NI/NF/ $f(x)$ & 0 \\
2 & $29 / 30 / 7.778992 e-9$ & $42 / 45 / 0.381 e-6$ & 0 \\
3 & $9 / 10 / 1.450669 e-5$ & $46 / 48 / 7.091824 e-7$ & $18 / 20 / 0.679 e-6$ & $32 / 34 / 1.9522245$ \\
4 & $9 / 10 / 1.9522245$ & $11 / 13 / 6.735123 e-5$ & $14 / 16 / 2.0000000$ & 2.0 \\
5 & $4 / 9 / 2.000009$ & $12 / 14 / 1.952225$ & $17 / 19 /-3.0000000$ & -3 \\
6 & $3 / 4 /-2.999949$ & $2 / 6 / 2.000098$ & $13 / 15 / 7.2000015$ & -1.20 \\
7 & $11 / 12 / 7.200000$ & $4 / 6 /-2.999866$ & $11 / 12 /-1.4142136$ & -1.0 \\
8 & $3 / 4 /-1.4142136$ & $10 / 12 / 7.200011$ & $66 / 68 /-0.9999994$ & -1.0 \\
9 & $9 / 10 /-0.9999638$ & $2 / 3 /-1.414214$ & $13 / 15 /-1.0000000$ & -8.0 \\
10 & $12 / 13 /-0.9999978$ & $4 / 6 /-0.9919815$ & $43 / 46 /-8.0000000$ & -44 \\
11 & $5 / 6 /-7.999999$ & $20 / 23 /-0.9999925$ & $43 / 45 /-43.999999$ \\
12 & $6 / 7 /-43.99797$ & - & $27 / 29 / 22.600162$ \\
\hline
\end{tabular}

\section{Conflict of Interests}

The author declares that there is no conflict of interests regarding the publication of this paper.

\section{Acknowledgments}

The author would like to thank the anonymous referees for their valuable comments and suggestions which help a lot to improve the paper greatly. The author also thanks Professor Gong-lin Yuan for his kind offer of the source BB codes on nonsmooth problems. This work is supported by the National Natural Science Foundation of China (Grant no. 11161003).

\section{References}

[1] J. B. Hiriart-Urruty and C. Lemaréchal, Convex Analysis and Minimization Algorithms, Springer, Berlin, Germany, 1993.

[2] M. Fukushima and L. Qi, "A globally and superlinearly convergent algorithm for nonsmooth convex minimization," SIAM Journal on Optimization, vol. 6, no. 4, pp. 1106-1120, 1996.

[3] L. Q. Qi and J. Sun, “A nonsmooth version of Newton's method," Mathematical Programming, vol. 58, no. 3, pp. 353-367, 1993.

[4] F. H. Clarke, Optimization and Nonsmooth Analysis, Wiley, New York, NY, USA, 1983.

[5] S. Lu, Z. Wei, and L. Li, "A trust region algorithm with adaptive cubic regularization methods for nonsmooth convex minimization," Computational Optimization and Applications, vol. 51, no. 2, pp. 551-573, 2012.
[6] L. Q. Qi, "Convergence analysis of some algorithms for solving nonsmooth equations," Mathematics of Operations Research, vol. 18, no. 1, pp. 227-244, 1993.

[7] R. Correa and C. Lemaréchal, "Convergence of some algorithms for convex minimization," Mathematical Programming, vol. 62, no. 1-3, pp. 261-275, 1993.

[8] M. Fukushima, "A descent algorithm for nonsmooth convex optimization," Mathematical Programming, vol. 30, no. 2, pp. 163-175, 1984.

[9] J. R. Birge, L. Qi, and Z. Wei, "Convergence analysis of some methods for minimizing a nonsmooth convex function," Journal of Optimization Theory and Applications, vol. 97, no. 2, pp. 357-383, 1998.

[10] Z. Wei, L. Qi, and J. R. Birge, "A new method for nonsmooth convex optimization," Journal of Inequalities and Applications, vol. 2, no. 2, pp. 157-179, 1998.

[11] N. Sagara and M. Fukushima, "A trust region method for nonsmooth convex optimization," Journal of Industrial and Management Optimization, vol. 1, no. 2, pp. 171-180, 2005.

[12] G. Yuan, Z. Wei, and Z. Wang, "Gradient trust region algorithm with limited memory BFGS update for nonsmooth convex minimization," Computational Optimization and Applications, vol. 54, no. 1, pp. 45-64, 2013.

[13] G. Yuan and Z. Wei, "The Barzilai and Borwein gradient method with nonmonotone line search for nonsmooth convex optimization problems," Mathematical Modelling and Analysis, vol. 17, no. 2, pp. 203-216, 2012.

[14] G. Yuan, Z. Wei, and G. Li, "A modified Polak-Ribière-Polyak conjugate gradient algorithm for nonsmooth convex programs," Journal of Computational and Applied Mathematics, vol. 255, pp. 86-96, 2014. 
[15] Q. Li, "Conjugate gradient type methods for the nondifferentiable convex minimization," Optimization Letters, vol. 7, no. 3, pp. 533-545, 2013.

[16] L. Han, G. Yu, and L. Guan, "Multivariate spectral gradient method for unconstrained optimization," Applied Mathematics and Computation, vol. 201, no. 1-2, pp. 621-630, 2008.

[17] G. Yu, S. Niu, and J. Ma, "Multivariate spectral gradient projection method for nonlinear monotone equations with convex constraints," Journal of Industrial and Management Optimization, vol. 9, no. 1, pp. 117-129, 2013.

[18] Z. Yu, J. Sun, and Y. Qin, "A multivariate spectral projected gradient method for bound constrained optimization," Journal of Computational and Applied Mathematics, vol. 235, no. 8, pp. 2263-2269, 2011.

[19] Y. Xiao and Q. Hu, "Subspace Barzilai-Borwein gradient method for large-scale bound constrained optimization," Applied Mathematics and Optimization, vol. 58, no. 2, pp. 275290, 2008.

[20] H. Zhang and W. W. Hager, "A nonmonotone line search technique and its application to unconstrained optimization," SIAM Journal on Optimization, vol. 14, no. 4, pp. 1043-1056, 2004.

[21] L. Lukšan and J. Vlček, "Test problems for nonsmooth unconstrained and linearly constrained optimization," Tech. Rep. 798, Institute of Computer Science, Academy of Sciences of the Czech Republic, Praha, Czech Republic, 2000.

[22] L. Lukšan and J. Vlček, "A bundle-Newton method for nonsmooth unconstrained minimization," Mathematical Programming, vol. 83, no. 3, pp. 373-391, 1998. 


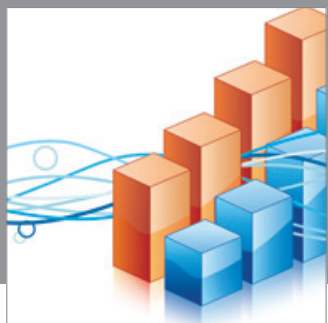

Advances in

Operations Research

mansans

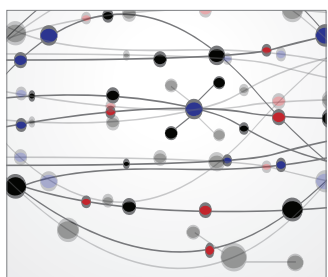

The Scientific World Journal
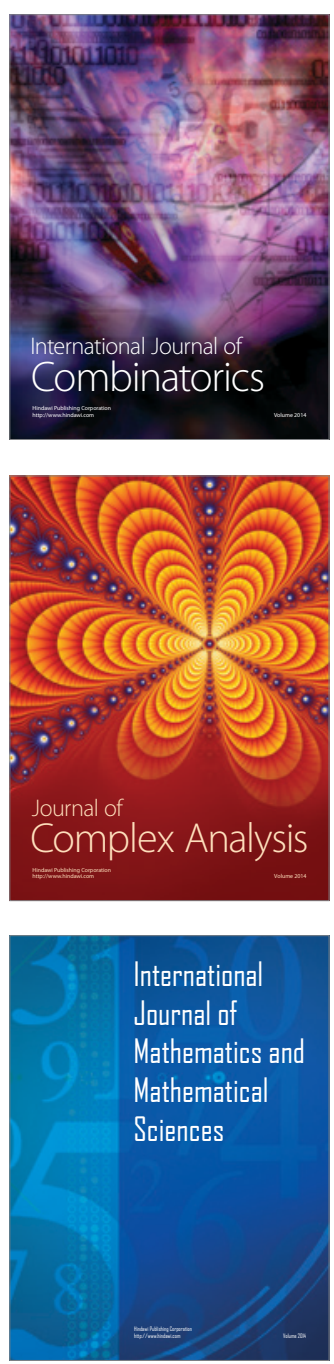
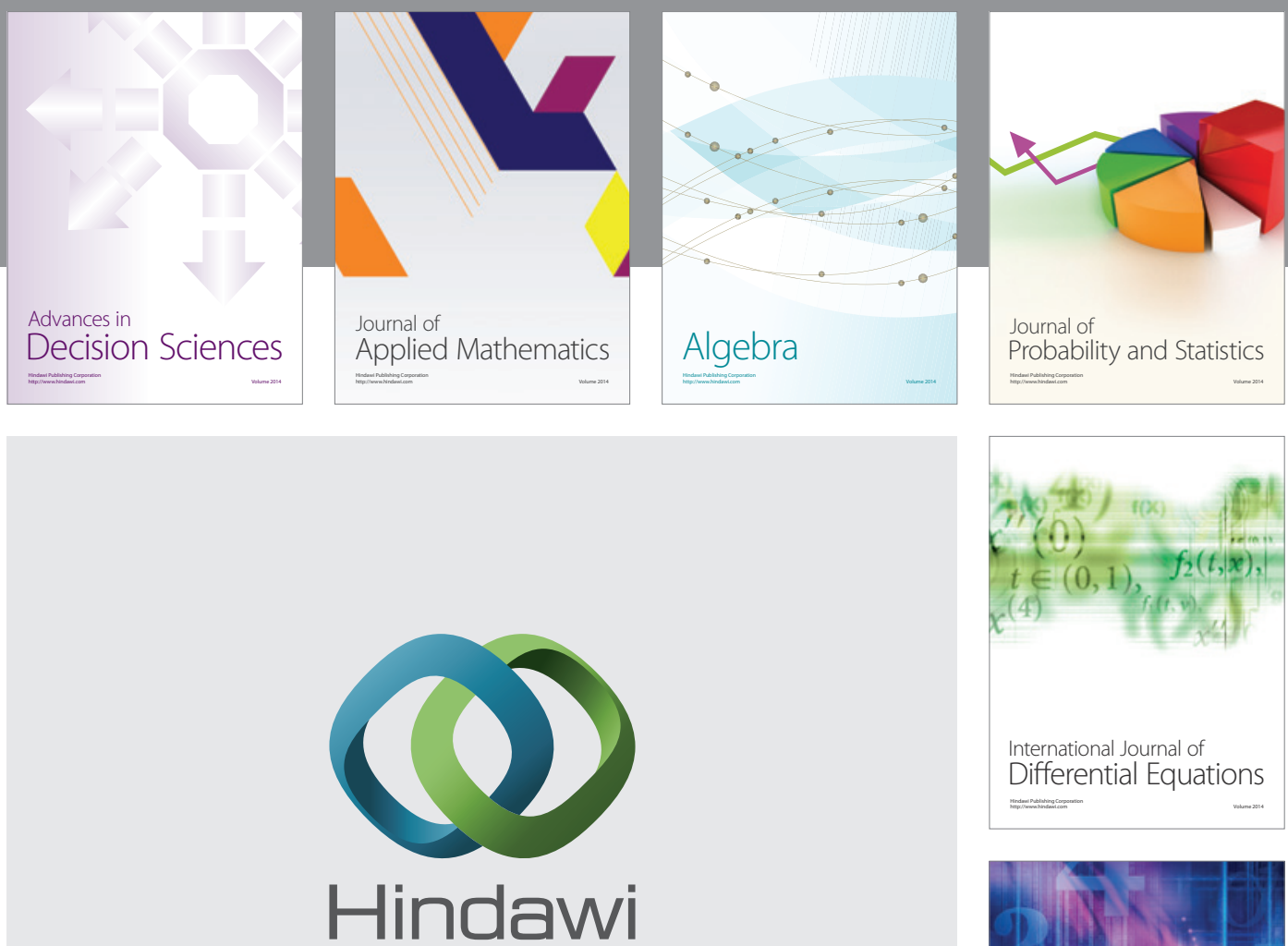

Submit your manuscripts at http://www.hindawi.com
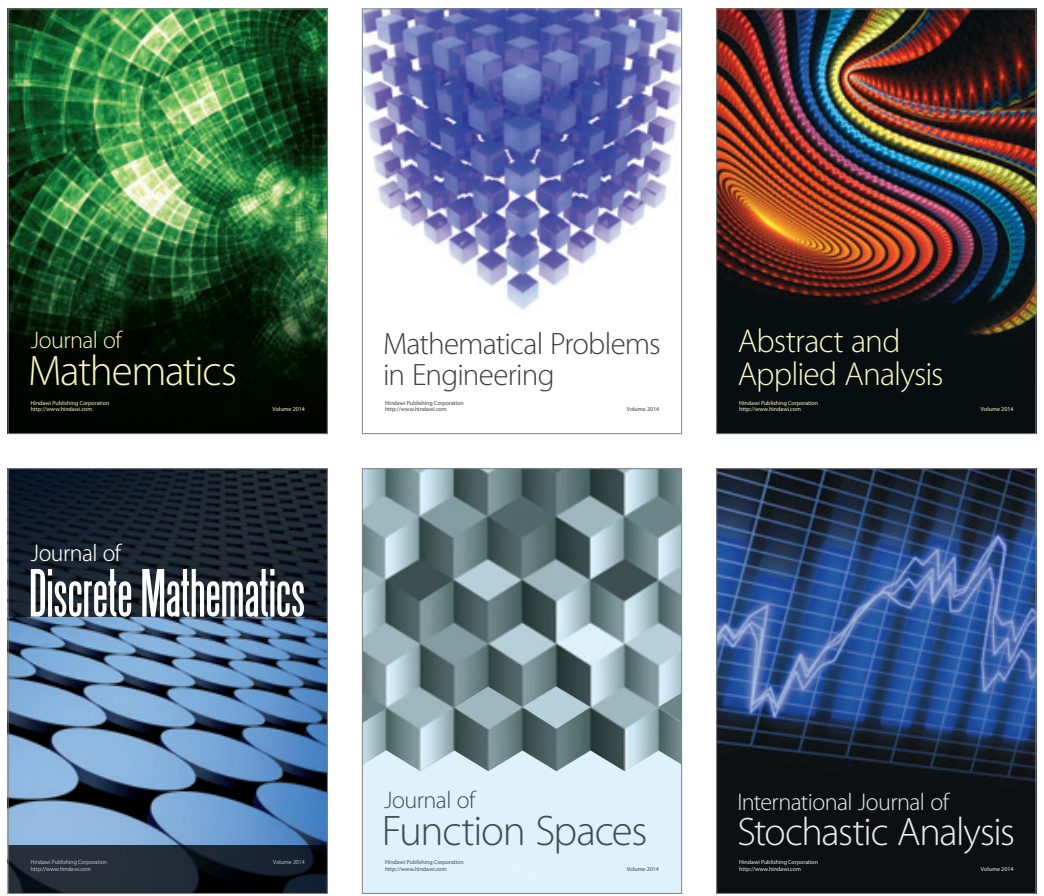

Journal of

Function Spaces

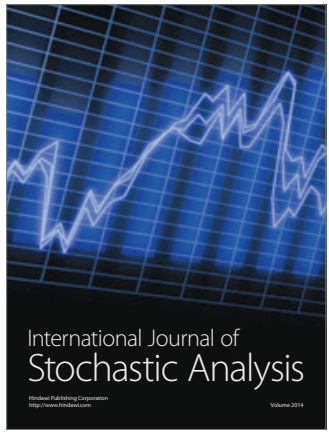

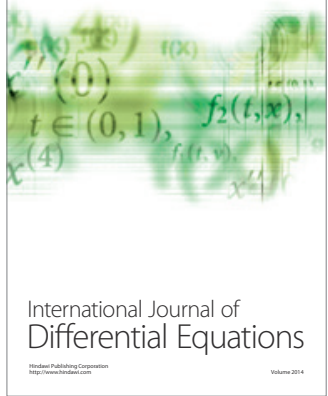
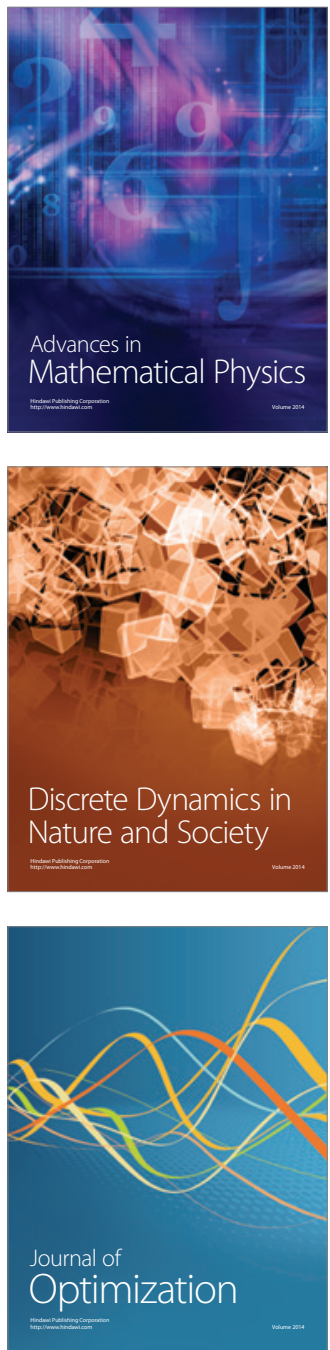